\title{
Experimental evidence of non-ideal compressible effects in expanding flow of a high molecular complexity vapor
}

\author{
Andrea Spinelli ${ }^{1}$ - . Giorgia Cammi ${ }^{1}$ S Simone Gallarini ${ }^{1} \cdot$ Marta Zocca ${ }^{2} \cdot$ Fabio Cozzi ${ }^{1} \cdot$ Paolo Gaetani ${ }^{1}$. \\ Vincenzo Dossena ${ }^{1} \cdot$ Alberto Guardone $^{2}$
}

Received: 3 November 2017 / Revised: 9 May 2018 / Accepted: 18 June 2018 / Published online: 16 July 2018

(c) The Author(s) 2018

\begin{abstract}
Supersonic expansions of a molecularly complex vapor occurring within the non-ideal thermodynamic region in the close proximity of liquid-vapor saturation curve were characterized experimentally for the first time. Results for two planar converging-diverging nozzles in the adapted regime and at different inlet conditions, from highly non-ideal to dilute gas state, are reported. Measurements of upstream total pressure and temperature are performed in the plenum ahead of the nozzle, while static pressure and supersonic Mach number measurements are carried out along the nozzle centerline. The investigated expansions are of interest for both fundamental research on non-ideal compressible flows and industrial applications, especially in the energy field. Siloxane MDM (octamethyltrisiloxane, $\mathrm{C}_{8} \mathrm{H}_{24} \mathrm{O}_{2} \mathrm{Si}_{3}$ ), a high molecular complexity organic compound, is used. Local pressure ratio $P / P_{\mathrm{T}}$ and Mach number $M$ measurements display a dependence on the inlet total state, a typical non-ideal feature different from dilute gas conditions.
\end{abstract}

\section{Introduction}

Non-ideal compressible flows of molecularly complex vapors are of interest for diverse industrial applications such as transportation of high-pressure fuels and chemicals and for turbomachines. The latter are typically implemented in chemical processes and in energy systems, as it is the case of compressors for refrigeration industry and turbo-expanders for organic Rankine cycles (ORCs). Working fluids involved are typically organic compounds such as aliphatic and aromatic hydrocarbons, halocarbons, and siloxanes, which feature high molecular complexity and high molecular mass. Expansions taking place within ORC turbine blade channels are of particular interest, since they occur in the close proximity of vapor saturation curve and critical point (Macchi

Electronic supplementary material The online version of this article (https://doi.org/10.1007/s00348-018-2578-0) contains supplementary material, which is available to authorized users.

Andrea Spinelli

andrea.spinelli@polimi.it

1 Energy Department, Politecnico di Milano, Via Lambruschini 4, 21056 Milan, Italy

2 Department of Aerospace Science and Technology, Politecnico di Milano, Via La Masa 34, 21056 Milan, Italy and Astolfi 2017; Colonna et al. 2015, 2008) thus entailing remarkable deviation from the ideal-gas behavior. Indeed, in these so-called non-ideal regions, the behavior of isentropic expansions is dependent on the flow stagnation conditions and not only on the expander geometry, as it is the case of ideal gases. This can be observed by analyzing the flow in terms of local Mach number $M$ and static-to-total ratio of local thermodynamic quantities, such as, for instance, pressure, temperature and density; thus obtaining ratios such as $P / P_{\mathrm{T}}, T / T_{\mathrm{T}}, \rho / \rho_{\mathrm{T}}$, where $P, T, \rho$ are, respectively, pressure, temperature and density, and the subscript $\mathrm{T}$ refers to total conditions. Moreover, departure from ideality can be so large to possibly lead to uncommon phenomena, such as non-monotonic variation of Mach number along isentropic expansions (Cramer and Fry 1993; Kluwick 2004), increase of the Mach number past oblique shock waves (Gori et al. 2017a), Vimercati et al. (2017) or even to non-classical ones, such as the occurrence of rarefaction shock waves (Thompson and Lambrakis 1973). These effects are ascribed to the action of long-range attractive and short-range repulsive intermolecular forces (Colonna and Guardone 2006) which make possible an unconventional increase of the speed of sound $c$ along isentropic expansion. The fundamental derivative of gas dynamics $\Gamma$ (Thompson 1971) is a measure of the change of speed of sound along isentropic expansions and therefore of non-ideal effects; it is defined as follows: 
$\Gamma=1+\frac{\rho}{c}\left(\frac{\partial c}{\partial \rho}\right)_{s}$

where the partial derivative is taken at constant specific entropy per unit mass $s$. $\Gamma$ provides a comprehensive description of the gas dynamic regimes. In the ideal-gas state, $P v=R T$, with $v$ the specific volume and $R$ the gas constant, and under the polytropic assumption of constant specific heats, $\Gamma=(\gamma+1) / 2>1$, where $\gamma>1$ is the specific heat ratio. Current models predict that, for molecularly complex fluids, a thermodynamic region exists in the vapor phase where $\Gamma<1$ and the flow behavior is non-ideal; if sub-regions of negative $\Gamma$ are also present, the regime is non-classical and expansion shock waves are also admissible. A detailed discussion on different compressible fluid regimes in quasi one-dimensional nozzle expansions is given in Guardone and Vimercati (2016).

The understanding of the fluid-dynamics of highly nonideal compressible flows is crucial for the appropriate design of devices operating in such non-ideal conditions. Therefore, detailed experimental data are needed about Non-Ideal Compressible Fluid Dynamics (NICFD) flows, with a particular focus on ORC power systems, which are unavailable in the open literature up to date. NICFD is the branch of fluid-dynamics dealing with compressible flows for which $P v \neq R T$.

To fill this gap, experiments are performed for different flow conditions on the Test Rig for Organic VApors (TROVA) (Spinelli et al. 2010; Pini 2011; Spinelli et al. 2013), the blow-down facility of the Laboratory of Compressible fluid dynamics for Renewable Energy Application (CREA Lab) of Politecnico di Milano. The TROVA test section is currently equipped with a modular planar nozzle, whose contoured profiles are specifically designed for the fluid and the operating conditions under scrutiny. However, linear turbine blade cascades can also be accommodated. A converging-diverging nozzle was selected as the simplest geometry expanding the vapor from subsonic to supersonic velocity, similarly to turbine blade channels. In addition, a straight axis nozzle permits the flow characterization by means of pressure taps and transducers with no adoption of directional probes, which are currently not available for organic vapor flows.

The results of an extensive experimental campaign are presented here for the first time. Siloxane MDM (octamethyltrisiloxane, $\mathrm{C}_{8} \mathrm{H}_{24} \mathrm{O}_{2} \mathrm{Si}_{3}$ ) was chosen as working fluid, since it is complex enough to exhibit a highly non-ideal behavior in the vapor phase (Colonna et al. 2007) and it is also widely applied in ORC power systems. The studied expansions are provided by two different nozzles, both designed to obtain a uniform outlet flow with Mach number of about 2 and 1.5 respectively, see Guardone et al. (2013).
The vapor flow is characterized by measuring the total pressure $P_{\mathrm{T}}$ and the total temperature $T_{\mathrm{T}}$ within a plenum upstream of the nozzle and the wall static pressure $P$ along the nozzle center line. The schlieren technique is also applied to visualize the density gradient field within the flow. Each nozzle is tested at variable inlet conditions, from highly non-ideal to almost ideal-gas state, as it is pointed out by the compressibility factor $Z=P v / R T$ varying between 0.63 and 0.99. As the fundamental derivative of gas dynamics, the compressibility factor is a measure of the flow non-ideality (being at the constant value of $Z=1$ for an ideal gas) and it is the parameter typically employed to remark deviations from the ideal gas behavior. During a single test run, the vapor is blown from an upstream reservoir which empties, thus feeding the nozzle at reducing pressure, allowing to explore a wide range of thermodynamic states.

Several tests are carried out in similar conditions, obtaining consistent results, thus proving the test repeatability. For four tests, out of eleven significant ones, measured data are thoroughly discussed, highlighting non-ideal compressible effects of different intensity by comparison with the nozzle flow field obtained in the ideal-gas state. The influence of inlet conditions, a distinctive feature of NICFD, is also pointed out. The flow features are qualitatively in accordance with those predicted by the quasi-one-dimensional theory for a polytropic van der Waals fluid and with those resulting from more accurate two-dimensional computational fluid dynamics (CFD) calculation using state-of-the-art thermodynamic models. For one of the two nozzles, a comparison is also performed with results obtained operating the nozzle with air, thus assessing the influence of different gas complexity on the polytropic ideal-gas flow.

The paper is organized as follows. Section 2 presents the TROVA facility and the measurement techniques employed, while the test procedure and conditions are described in Sect. 3. Section 4 discusses the data consistency and their representation in time and space. The results of the investigation are presented in Sect. 5 by discussing the nozzle flow features at selected inlet conditions, characterized by different non-ideal behavior of the vapor. Finally, Sect. 6 draws some conclusions.

\section{Experimental facility and measurement techniques}

The TROVA facility is a blow-down wind tunnel built to investigate NICFD flows, especially for ORC applications. The working fluid is first isochorically heated in a high-pressure vessel (HPV) up to saturated, superheated, or supercritical conditions. The vapor is then discharged by the HPV, expands from subsonic to supersonic velocity through the converging-diverging nozzle and it is then collected in a 
low-pressure vessel (LPV). Here, the vapor is condensed and pumped back to the HPV by means of a membrane pump. The nozzle total pressure $P_{\mathrm{T}}$, thus the discharged flow rate, is controlled by a fast operating main control valve (MCV). Extensive details about the test rig and its design can be found in Spinelli et al. $(2010,2013)$.

\subsection{Test section and instrumentation}

The TROVA was designed to investigate a wide variety of organic fluids at different operating conditions. The nozzle test section is modular and allows to accommodate straightaxis converging-diverging nozzles with a rectangular cross section, specifically designed for the fluid and the operating conditions under scrutiny, see Guardone et al. (2013). The straight axis nozzle allows to characterize the pressure field at the flow core by applying the isentropic hypothesis, thus avoiding the use of single or multi-hole directional probes, which would require fluid-specific aerodynamic calibration. This hypothesis does not apply if shock waves are observed in the diverging portion. Furthermore, the planar configuration permits an easy implementation of the optical access.

Two nozzles were tested, both designed to deliver a uniform flow at the exit section:

- Nozzle M2.0 provides an exit flow with design Mach number $M=2$ and features a backward facing step at the geometrical throat, machined to fix the throat position independent of any possible unsteadiness of the boundary layer. In addition, the recessed step allowed to investigate the shock structure originating downstream the separated region. The step non-dimensional height is $h / H=0.012$, where $H$ is the nozzle semi-height at throat. Details are given in Gori et al. (2017b).

- Nozzle M1.5 is designed, with a clean geometry, to deliver an exit flow at Mach number $M=1.5$. No step was machined, since the throat position revealed stable, as pointed out by non-fluctuating pressure signals. An increased roughness $(\mathrm{Ra} \approx 0.6 \mu \mathrm{m})$ was adopted at the contoured end-walls to form Mach waves, whose slope, detected through the schlieren images, provides a direct measurement of the local Mach number.

The diverging portion of each nozzle has been designed by using the method of characteristics coupled with state-ofthe-art thermodynamic models for siloxane MDM (Guardone et al. 2013; Lemmon et al. 2013), while the converging part has been designed using a 5 th order polynomial profile.

Along the nozzle axis, nine pressure taps of $0.3 \mathrm{~mm}$ diameter are machined on the rear steel plate. They are conveniently labeled with increasing numbers, from 1 to 9 in the streamwise direction, see Fig. 1, and congruent labeling is adopted for the correspondent measured pressures. Each

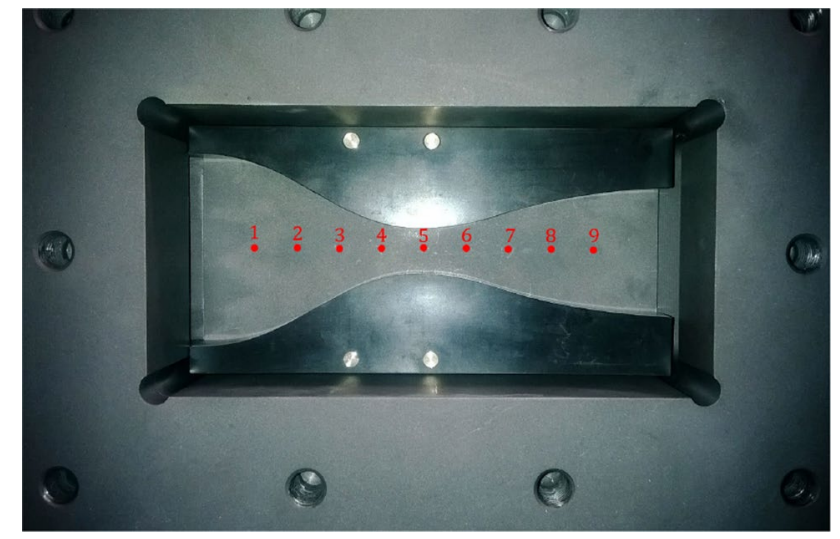

Fig. 1 Static pressure tap location on the rear plate along the nozzle axis. The flow is from left to right. In this picture the plate is not mirror polished

tap is connected, through a $30 \mathrm{~mm}$ long line-cavity system, to a pressure transducer, which measures wall static pressure $P$. The capacity connecting the flow with the sensing element exhibits a volume of about $130 \mathrm{~mm}^{3}$, leading to a resonance frequency of line-cavity system of the order of $200 \mathrm{~Hz}$, estimated by a viscous model developed for an arbitrary complex system of lines and cavities, see Antonini et al. (2008). During a test run not all the nozzle taps are necessarily active, due to possible unavailability of pressure sensors with the proper full scale; however, flow field regions interested by high pressure gradients are always covered. The rear plate is mirror polished to perform double passage schlieren, while the frontal optical access is provided by a planar quartz window.

Stagnation conditions $P_{\mathrm{T}}, T_{\mathrm{T}}$ are measured in the settling chamber ahead the test section, using a wall pressure transducer (due to the very low local flow velocity of about $1 \mathrm{~m} / \mathrm{s}$ ) and two thermocouples, TCK and TCJ, of K and J type respectively. Their hot junctions, whose diameter is of $0.25 \mathrm{~mm}$ (K-type) and $0.7 \mathrm{~mm}$ (J-type), are located at the chamber axis and at a relative distance of about $200 \mathrm{~mm}$ streamwise. The time constants of the considered thermocouples were estimated using the two sensor characterization method proposed by Hung et al. (2005). The analysis yields a time constant of $\tilde{\tau}_{\mathrm{TCK}}=0.28 \mathrm{~s}$ and $\tilde{\tau}_{\mathrm{TCJ}}=1.34 \mathrm{~s}$ for the $\mathrm{K}$ and $\mathrm{J}$ thermocouples respectively. Furthermore, due to the low frequency content of the physical signal (only $0.005 \%$ of the signal energy is found above $0.2 \mathrm{~Hz}$, if the total pressure signal is taken as reference), the dynamic temperature reconstruction procedure yields an input signal always lying well within the sensor uncertainty. Compensation is therefore not required.

Pressure sensors are miniaturized piezo-resistive transducers (sensing element diameter of $3.8 \mathrm{~mm}$ ), operating up to $343{ }^{\circ} \mathrm{C}$ and exhibiting high natural frequency $\left(\sim 10^{2}\right.$ to 
$\sim 10^{3} \mathrm{kHz}$ depending on the full scale), thus not limiting the pressure measurement response. Due to their considerable sensitivity to temperature variation, they were calibrated both in pressure and temperature in the range of 1 bar to full scale ( $3.5 \leq \mathrm{FS} \leq 40$ bar) and of $25-300{ }^{\circ} \mathrm{C}$ respectively. The two thermocouples were calibrated in the same temperature range $\left(25-300^{\circ} \mathrm{C}\right)$. The final accuracy of each sensor is summarized in Table 1 . The data acquisition (DAQ) system consists of analog modules for signal conditioning and of a high speed 16 bit ADC data acquisition board.

Continuous imaging of the density gradient field is provided by schlieren visualization, to complement the discrete pressure measurements at the nozzle axis. The system is of double passage type, with the emitting and receiving components on the same optical bench, featuring the advantage of easy alignment and of enhanced sensitivity. A high-speed CMOS camera is digitally triggered to synchronize the schlieren images with the pressure and temperature data. The camera resolution is $1936 \times 1216$ pixels, with a pixel dimension of $5.86 \mu \mathrm{m}$. The knife orientation of the system is normal to the nozzle centerline, thus allowing to visualize density gradients along the nozzle axis direction. In addition, its position is such that compression waves (positive density gradients) appear as dark regions, while expansion waves as bright ones. The light source is a $100 \mathrm{~W} \mathrm{Hg}$ arc-lamp. Further details on the data acquisition system and on the schlieren bench are given in Spinelli et al. (2016) and Cozzi et al. (2015).

\section{Test description and operating conditions}

For each of the two nozzles, M2.0 and M1.5, several tests were carried out on the expanding flow of MDM vapor, starting either at moderate or at high non-ideal thermodynamic conditions, in subcritical and slightly superheated state, thus close to the saturation curve. Tests performed at moderate non-ideal states feature initial total pressure and temperature of about $P_{\mathrm{T}}=4.5$ bar and $T_{\mathrm{T}}=240{ }^{\circ} \mathrm{C}$, namely, reduced conditions of $P_{\mathrm{Tr}}=P_{\mathrm{T}} / P_{\mathrm{C}}=0.31$ and $T_{\mathrm{Tr}}=T_{\mathrm{T}} / T_{\mathrm{C}}=0.91$, where $P_{\mathrm{C}}$ and $T_{\mathrm{C}}$ are, respectively, the pressure and the temperature at the critical point. Correspondingly, the minimum value of the compressibility factor at total conditions $Z_{\mathrm{T}}$ is around 0.81 . The value of $Z_{\mathrm{T}}$ is calculated as a function of $P_{\mathrm{T}}$ and $T_{\mathrm{T}}$ by using the Helmoltz energy approach of Span and Wagner (2003) implemented in RefProp for MDM. Tests at high non-ideal conditions are carried out starting from stagnation pressure and temperatures of about $P_{\mathrm{T}}=9 \mathrm{bar}$ $\left(P_{\mathrm{Tr}}=0.63\right)$ and $T_{\mathrm{T}}=270{ }^{\circ} \mathrm{C}\left(T_{\mathrm{Tr}}=0.96\right)$, with compressibility factors as low as $Z_{\mathrm{T}}=0.63$. Four out of eleven tests (selected within the overall experimental campaign) are discussed in detail in this paper. Indeed, different tests performed at similar (though not identical) inlet conditions provided comparable results; therefore the four selected tests are taken as representative of the two tested nozzles and of the two initial levels of pressure (high and low) corresponding to high and moderate non-ideality. Accordingly, the four tests are labeled using the nozzle name, M2.0 or M1.5, and the pressure level, high $(\mathrm{H})$ or low $(\mathrm{L})$. For this reference experiments, Table 2 summarizes the operating conditions at time $t=0 \mathrm{~s}$, when the total compressibility factor $Z_{\mathrm{T}}$ is at minimum value.

A single test is triggered by the opening of the main control valve (MCV) and the consequent vapor discharge through the nozzle. For the entire test, the valve is kept at a fixed $100 \%$ opening position, therefore the upstream total pressure is not controlled, entailing the opportunity of exploring a wide variety of thermodynamic states during one single run. Indeed, after the opening transient of about $2 \mathrm{~s}$, the HPV empties and $P_{\mathrm{T}}$ decreases; starting from non-ideal dense-vapor conditions, more dilute states are encountered as the test proceeds and the ideal gas state is approached.

The relaxation time of the nozzle is more than two order of magnitude lower than the one related to the emptying process of the HPV (Spinelli et al. 2010, 2013) therefore, a steady-state nozzle flow can be assumed at any fixed time. Indeed, data are acquired at a high frequency, which is $1 \mathrm{kHz}$ for pressure and temperature measurements, while the exposure time for schlieren imaging is set to $1 \mathrm{~ms}$. Due to the extremely low initial pressure within the LPV, which is below 10 mbar, and the large LPV-to-HPV capacity ratio (about 6), the test section operates in under-expanded conditions during the entire run and an isentropic flow can be assumed within the large nozzle core, except in the very

Table 1 Type, calibration range and expanded uncertainty (U) of pressure and temperature sensors

\begin{tabular}{lllllc}
\hline Property & Sensor & Type & Calibration range $P($ bar $)$ & $\begin{array}{l}\text { Calibration } \\
\text { range } T\left({ }^{\circ} \mathrm{C}\right)\end{array}$ & $U_{P}(\% \mathrm{FS})$ \\
\hline$T_{\mathrm{T}}$ & Thermocouple & $\mathrm{K}(\mathrm{Ni} / \mathrm{Cr}-\mathrm{Ni} / \mathrm{Al})$ & - & $25-300$ & - \\
$T_{\mathrm{T}}$ & Thermocouple & $\mathrm{J}(\mathrm{Fe}-\mathrm{Cu} / \mathrm{Ni})$ & - & $25-300$ & - \\
$P_{\mathrm{T}}, P$ & Piezo-resistive transducer & Kulite $\mathrm{XTEH}$ & $1-\mathrm{FS}(3.5-40)$ & $25-300$ & $\sim 0.07$ \\
\hline
\end{tabular}

$\mathrm{FS} \in[3.5,40 \mathrm{bar}]$ is the transducer full scale 
close proximity of the walls. This hypothesis is also confirmed by CFD simulations, see Gori et al. (2017b).

For the four analyzed tests, Fig. 2a depicts the steady state, isentropic expansions processes occurring within the nozzles at time $t=0 \mathrm{~s}$, in the temperature-specific entropy $(T-s)$ diagram. Figure $2 \mathrm{~b}$ shows the time evolution of the inlet condition $\left(P_{\mathrm{T}}, T_{\mathrm{T}}\right)$ during each test. In both figures, the contour of $Z$ is reported, which increases towards the $Z=1$ limit as $P_{\mathrm{T}}$ reduces. The specific entropy $s$ was calculated from $P_{\mathrm{T}}$ and $T_{\mathrm{T}}$ using the RefProp model for MDM.

A single test concludes as the nozzle becomes overexpanded, with shock waves entering the divergent portion. This condition occurs in extremely dilute gas states, which are of scarce interest. The test duration obviously depends on the run conditions and on the nozzle throat area; it is about 150 and $200 \mathrm{~s}$ for tests performed at high- and low-pressure level respectively.

\section{Data representation and consistency}

This section describes how the measured pressure, temperature and schlieren images are processed and represented. The acquired data are also complemented with the calculated values of the compressibility factor $Z$ and of the fundamental derivative of gas dynamics $\Gamma$, which both point out the level of flow non-ideality. In addition, the repeatability of the test procedure is assessed by comparing data set gathered during eleven different tests.

(a) Z

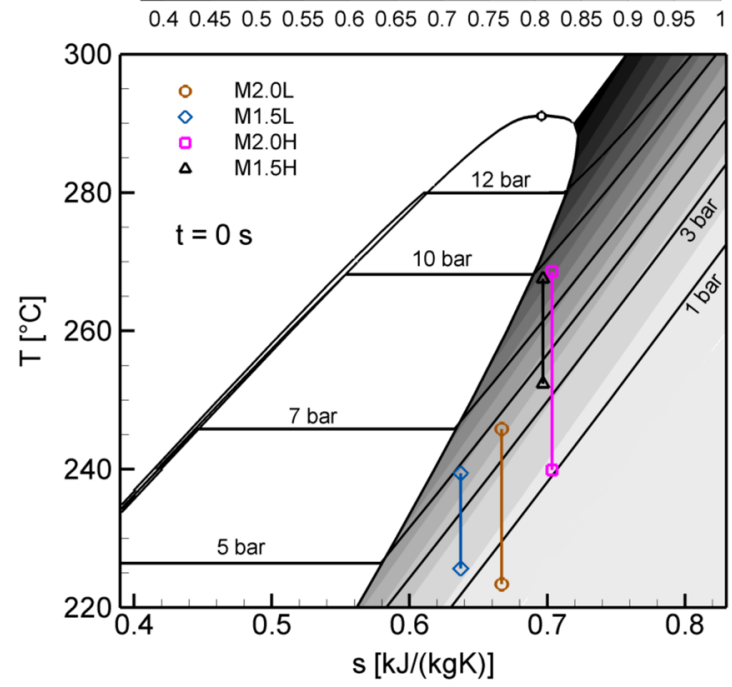

Fig. 2 a Nozzle expansions from total inlet conditions to static outlet conditions for the four representative tests $\mathrm{M} 2.0 \mathrm{~L}, \mathrm{M} 1.5 \mathrm{~L}, \mathrm{M} 2.0 \mathrm{H}$, $\mathrm{M} 1.5 \mathrm{H}$, at time $t=0 \mathrm{~s}$. Expansion processes are plotted in the temperature-specific entropy $(T-s)$ diagram. b Explored thermodynamic

\subsection{Data representation}

During each experiment run pressure and temperature signals are acquired at a sampling frequency of $1 \mathrm{kHz}$, while schlieren images are saved at a rate of 20 frames per second (fps) and with an exposure time $1 \mathrm{~ms}$. To synchronize pressure and temperature signals with schlieren images, pressure and temperature data are averaged in packages of 50 points, establishing a time resolution of $50 \mathrm{~ms}$ for the whole data set. Valve MCV starts opening at time $t=t_{\mathrm{s}} \approx-2 \mathrm{~s}$ and it is fully open at time $t=0 \mathrm{~s}$ which is set as the initial time of the experiment; correspondingly, the maximum total pressure $P_{\mathrm{T}}$ and the minimum total compressibility factor $Z_{T_{\min }}$ are reached upstream of the nozzle. The test final time $t_{\mathrm{f}}$ is marked by the occurrence of shock waves entering the diverging portion of the nozzle, which are detectable through the increasing of pressure measured from tap 9. Taking test M2.0L as an example, Fig. 3 a shows the time evolution of the total pressure $P_{\mathrm{T}}$, of the total temperature $T_{\mathrm{T}}$ and of wall static pressure $P$, measured at different locations along the nozzle axis during the experiment run. The test lasts approximately $195 \mathrm{~s}$ and static pressure signals are labeled according to the corresponding tap numbers, 1-9 streamwise, with tap number 5 located at the geometrical throat. For clarity, only signals of five taps $(3,5,6,7$ and 9$)$ out of the active eight ones are plotted. All pressure values decrease in time due the emptying of the HPV, while pressure along the nozzle reduces as the vapor expands. Contrarily, the total temperature $T_{\mathrm{T}}$ does not decrease monotonically with time

\section{(b) Z}
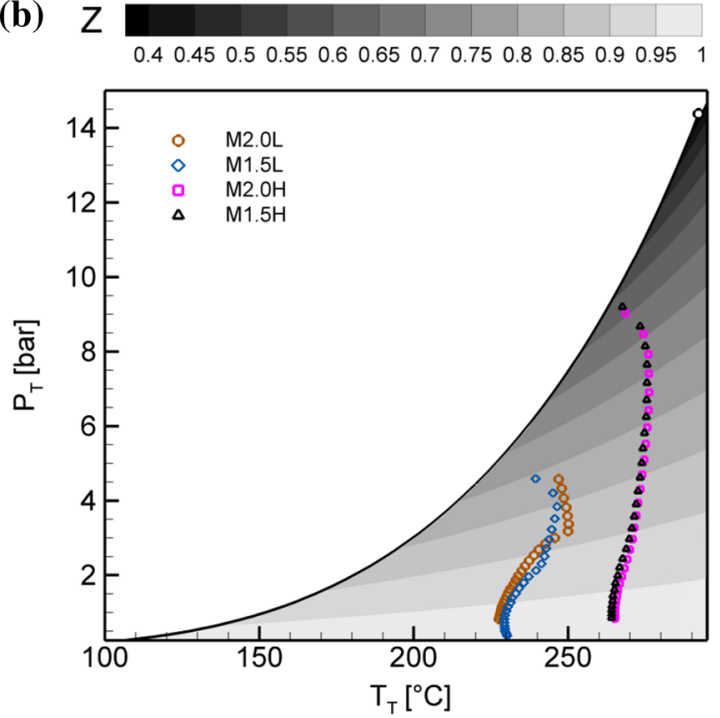

region in the pressure-temperature $(P-T)$ plane for the aforementioned tests for $t \geq 0$ and with a time step of $5 \mathrm{~s}$. Both the specific entropy $s$ and the compressibility $Z$ factor are calculated from the RefProp model of MDM 
Table 2 Operating conditions for reference experiments at the test start, $t=0 \mathrm{~s}$

\begin{tabular}{llllllll}
\hline Test & $\mathrm{P}_{\mathrm{T}}($ bar $)$ & $\mathrm{T}_{\mathrm{T}}\left({ }^{\circ} \mathrm{C}\right)$ & $\mathrm{P}_{\mathrm{Tr}}$ & $\mathrm{T}_{\mathrm{Tr}}$ & $\mathrm{Z}_{\mathrm{T}}$ & $\beta$ & $\mathrm{M}_{\text {out }}$ \\
\hline M2.0L & 4.58 & 247 & 0.32 & 0.92 & 0.82 & 9.83 & 2.10 \\
M1.5L & 4.59 & 239 & 0.32 & 0.91 & 0.81 & 3.34 & 1.55 \\
M2.0H & 9.02 & 269 & 0.63 & 0.96 & 0.65 & 9.16 & 2.03 \\
M1.5H & 9.20 & 268 & 0.64 & 0.96 & 0.63 & 3.12 & 1.50 \\
\hline
\end{tabular}

$P_{\mathrm{T}}, T_{\mathrm{T}}$ are total pressure and temperature and $P_{\mathrm{Tr}}, T_{\mathrm{Tr}}$ are their reduced values. $Z_{\mathrm{T}}$ is the total compressibility factor, $\beta$ the adapted expansion ratio and $M_{\text {out }}$ is the exit Mach number as expected. This is possibly due to non-uniformity of the temperature within the HPV and the heated pipe upstream of the plenum. Small fluctuations are visible during the initial part of test $\mathrm{M} 2.0 \mathrm{~L}$; they are of small amplitude (within the thermocouple uncertainty) and negligible in other tests. However, time instants corresponding to these fluctuations and the related data set are not considered among those extracted for steady nozzle flow analyses (see discussion below and Fig. 3b).

Figure $3 \mathrm{a}$ also reports the evolution of the compressibility factor $Z_{\mathrm{T}}$ and of the fundamental derivative of gas dynamics $\Gamma_{\mathrm{T}}$ in the plenum. Both values, which are calculated as a function of $P_{\mathrm{T}}$ and $T_{\mathrm{T}}$ from the RefProp model, provide an indication of the flow non-ideality at nozzle inlet. They are initially well below one and monotonically increase throughout the test, consistently with decreasing non-ideality until the ideal-gas state is approached at the run conclusion. Notice that for MDM, in the polytropic ideal gas (PIG) limit and in the investigated temperature range $\Gamma_{\mathrm{PIG}} \approx 1.009$. The

(a)

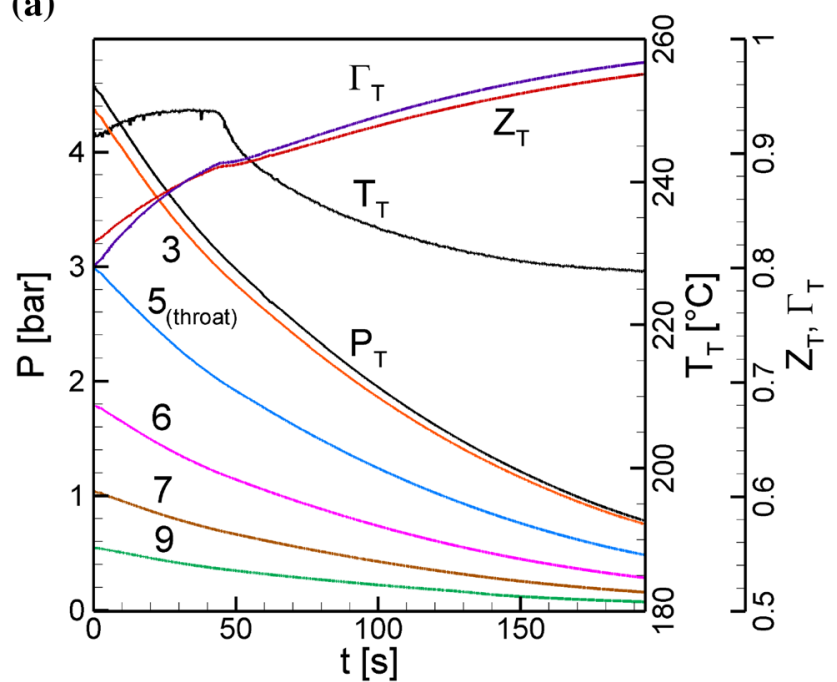

Fig. 3 a Time trends of measured $P_{\mathrm{T}}, T_{\mathrm{T}}$ and $P$ for test M2.0L. For plot clarity static pressure at the axis is only reported for taps 3,5 (geometrical throat), 6, 7, 9. The evolution of $Z_{\mathrm{T}}$ and $\Gamma_{\mathrm{T}}$, which are calculated using the RefProp model, is also shown to point out the non-ideal behavior of the flow. $\mathbf{b}$ Time trends of measured $P$ at each active pressure tap along the nozzle axis for test M2.0L. The noz- analyzed tests other than M2.0L feature very similar evolution and are therefore, not discussed in detail.

Figure $3 \mathrm{~b}$ depicts the time trend of pressure signals along the non-dimensional nozzle axial coordinate $x$, for test M2.0L. The $x$ coordinate is made non-dimensional by the nozzle semi-height at the geometrical throat $H$. Plotted signals extend to negative time since they include the opening transient of MCV. The three-dimensional plot clarifies that samples of the entire data set $P_{\mathrm{T}}, T_{\mathrm{T}}, P$ and schlieren images can be taken at different time in the range $\left[0, t_{\mathrm{f}}\right]$ (each equivalent to different $Z_{\mathrm{T}}$ in the range $\left[Z_{T_{\min }}, Z_{T_{\max }}\right]$ ) through cutting planes at different time $t$ ( $Z_{\mathrm{T}}$ in Fig. $3 \mathrm{~b}$ ). Each sample provides a steady-state nozzle flow field, with different level of non-ideality, marked by the value of $Z_{\mathrm{T}}$. Meaningful time instants for data extraction were chosen to cover the compressibility factor range with five, equally spaced, values. At each time, measured quantities are plotted against the axial coordinate, as it is standard practice in nozzle flows. In particular the local pressure ratios $P / P_{\mathrm{T}}$ and, for

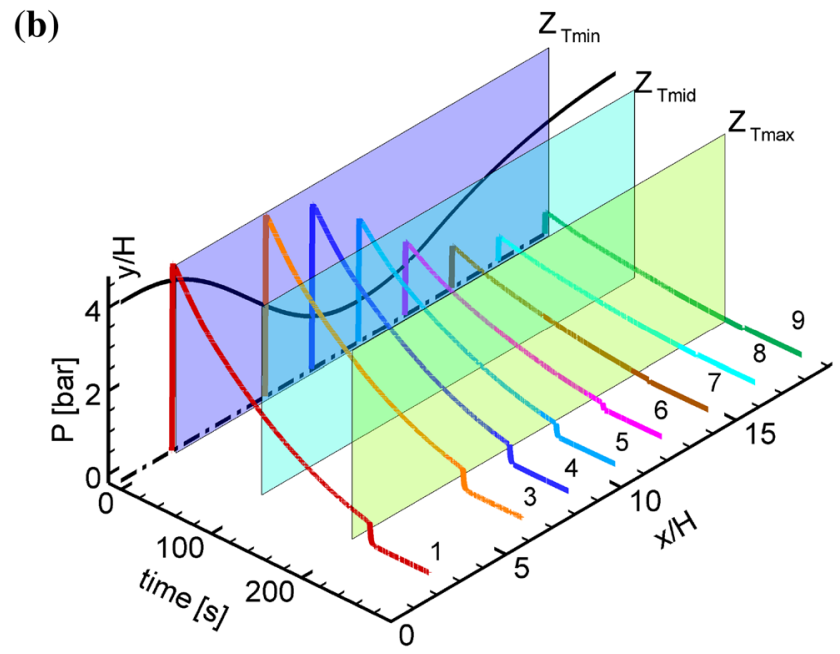

zle non-dimensional semi-profile (black line) is superimposed to the $P-x / H$ plane at the starting time $t_{\mathrm{s}} ; y / H$ represents the nondimensional coordinate normal to the axis. Steady-state sampling of the data set at three different time and $Z_{\mathrm{T}}$ values is also represented by three exemplifying cutting planes 
the diverging portion only, the local Mach number are discussed. These data are complemented with the local values of the compressibility factor $Z$, identifying flow non-ideality along the nozzle. Under the assumption of the boundary layer theory, the flow core is assumed to be isentropic and therefore $Z=Z(P, s)$ is calculated, using the RefProp model, as a function of the measured static pressure $P$ and of the specific entropy $s$, which is in turn computed from the stagnation conditions $s=s\left(P_{\mathrm{T}}, T_{\mathrm{T}}\right)$.

\subsection{Consistency of experimental data}

Consistency of experimental measures is now assessed. Eleven satisfactory experimental runs, performed using the two nozzles, are considered for consistency analysis and are reported in Table 3, together with total condition selected for the required comparison. They correspond to $Z_{T, \max }$ states, which are used to evaluate consistency of the whole data set. Different tests are labeled according to the execution sequence and include the four reference test (see Table 2).

The nozzle local pressure ratio $P / P_{\mathrm{T}}$ is the parameter of choice in this analysis, since it is obtained from measurements only, without employing the thermodynamic model. Therefore, the consistency of experimental measures is evaluated without the introduction of inaccuracies other than the instrument uncertainty. Furthermore, the local pressure ratio is a quantity which significantly characterizes isentropic nozzle flows; for instance in the PIG case, the local value of $P / P_{\mathrm{T}}$ in an adopted nozzle depends on the fluid and the geometry only.

To verify the test repeatability, a sufficiently large number of nozzle operating conditions $\left(P_{\mathrm{T}}, T_{\mathrm{T}}\right)$ are required for comparison, due to the non-ideal dependence of the nozzle flow on the stagnation conditions. Unfortunately, total pressure

Table 3 Tests considered for consistency analysis

\begin{tabular}{|c|c|c|c|c|}
\hline Nozzle & Test name & $P_{\mathrm{T}}(\mathrm{bar})$ & $T_{\mathrm{T}}\left({ }^{\circ} \mathrm{C}\right)$ & $Z_{T_{\text {max }}}$ \\
\hline \multirow[t]{5}{*}{ M2.0 } & Test04 $\equiv \mathrm{M} 2.0 \mathrm{~L}$ & 0.80 & 228 & 0.97 \\
\hline & Test06 & 0.21 & 236 & 0.99 \\
\hline & Test08 & 0.50 & 231 & 0.98 \\
\hline & Test09 & 0.73 & 237 & 0.97 \\
\hline & Test $10 \equiv \mathrm{M} 2.0 \mathrm{H}$ & 0.80 & 265 & 0.98 \\
\hline \multirow[t]{6}{*}{ M1.5 } & Test11 $\equiv \mathrm{M} 1.5 \mathrm{H}$ & 0.84 & 264 & 0.98 \\
\hline & Test12 & 0.30 & 233 & 0.99 \\
\hline & Test13 $\equiv \mathrm{M} 1.5 \mathrm{~L}$ & 0.52 & 230 & 0.98 \\
\hline & Test14 & 0.60 & 263 & 0.98 \\
\hline & Test16 & 0.22 & 263 & 0.99 \\
\hline & Test17 & 0.89 & 263 & 0.97 \\
\hline
\end{tabular}

The type of nozzle, the measured total pressure and total temperature are reported. The corresponding maximum total compressibility factors are also shown and temperature are rarely the same at any time in different tests, even when equal values of $Z_{\mathrm{T}}$ are encountered. This is due to the fact that the facility operation allows to set the test conditions by adjusting the total state at the HPV and not directly at nozzle inlet, and these two states are connected by a transient process which is not perfectly repeatable (see Spinelli et al. 2010, 2013). Therefore, discrepancies among measurements of different tests might be due to the different extent of non-ideal-gas effects and not to unreliability of the measurement system. However, a few similar conditions in terms of nozzle stagnation states are available for comparison of different tests (test $11,14,16$, and 17 according to labeling of Table 3$)$ at non-ideal conditions $\left(Z_{T}=0.70\right.$ for nozzle M1.5). Results are plotted in Fig. 4 in terms of pressure ratio distribution along the nozzle axis. The four tests considered share the same total compressibility factor and stagnation conditions which are bounded in a very limited range, about $3{ }^{\circ} \mathrm{C}$ in temperature and 0.15 bar in pressure. Moreover, test pairs 11 and 14 as well as 16 and 17 feature the same inlet nozzle condition, since they are within the limits defined by uncertainty bars. The measured pressure ratios at the nozzle axis are well overlapped, thus indicating repeatability of the measured data.

Furthermore, when the compressibility factor approaches unity, non-ideal effects weaken and measured pressure ratio values converge to the ones predicted by the dilute gas theory, which are independent from the upstream thermodynamic state. Thus, for further verification of measurements consistency, pressure ratios are compared at $Z_{T_{\max }}$, namely at the most ideal condition at which data are available for

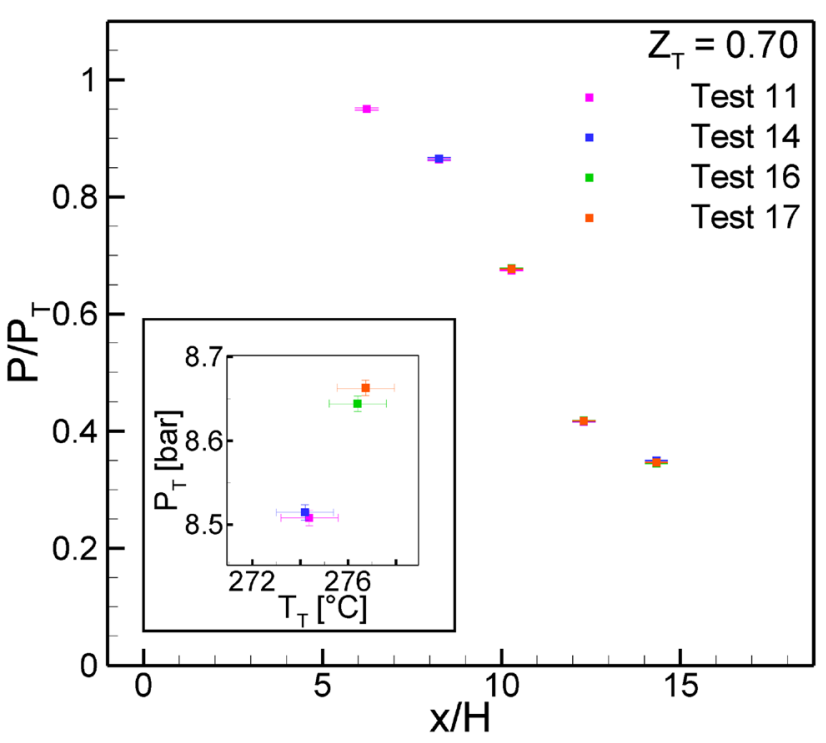

Fig. 4 Comparison of pressure ratio $P / P_{\mathrm{T}}$ distribution along the nozzle axis for tests $11,14,16$, and 17. Nozzle inlet stagnation conditions $P_{\mathrm{T}}$ and $T_{\mathrm{T}}$ are also reported for the data sets which are compared at the same total compressibility factor $Z_{\mathrm{T}}=0.70$ 
each experimental run, independently of the initial non-ideal state. The $Z$ values indicate the proximity to the ideal-gas conditions, while the polytropic hypothesis is supported by the limited temperature variation among the considered points (see Table 3), which entails negligible change in the ideal gas heat capacities. Experimental points from the whole set of 11 tests, complemented with uncertainty bars, are shown in Fig. 5. For both nozzles, the measured pressure ratios lie within the error bars from one another, thus proving the consistency of experimental data acquired during different experimental runs.

\section{Results and discussion}

This section presents detailed results obtained for the four tests representative of the whole experimental campaign. The gathered data are discussed, at variable non-ideal conditions, in terms of local pressure ratio $P / P_{\mathrm{T}}$ and (for the divergent portion only) of Mach number $M$ measured along the nozzle axis. Together with experimental points, calculated distributions of the compressibility factor $Z$ at the measuring point are provided to underline the non-ideal effect pertaining to each condition analyzed. For all conditions the flow at the centerline is assumed to be isentropic.

Nozzle expansions at different time and non-ideal conditions are analyzed; they refer to thermodynamics states selected so to obtain operating conditions with appreciable distinction in the levels of non-ideality. For each test five significant values of $Z_{\mathrm{T}}$ are therefore identified; they are the minimum value $Z_{T_{\min }}$, the maximum one $Z_{T_{\max }}$ and three intermediate values $Z_{\text {Tmid }, i}$, see Table 4 .

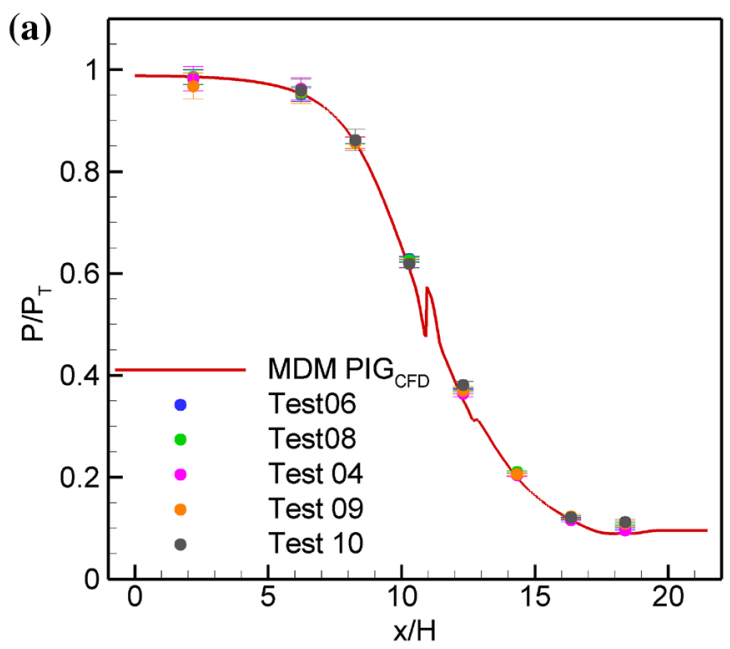

Fig. 5 Pressure ratio $P / P_{\mathrm{T}}$ trends along the nozzle axis for all tests on the two nozzles at respective $Z_{T_{\max }}$ conditions. For each geometry, results extracted at the axis line from two-dimensional CFD viscous simulation, performed in the PIG hypothesis, are reported for refer-
Table 4 Selected compressibility factor $Z_{\mathrm{T}}$ upstream of the nozzle for reference experiments

\begin{tabular}{llllll}
\hline Test & $Z_{T_{\min }}$ & $Z_{\text {Tmid, } 1}$ & $Z_{\text {Tmid, } 2}$ & $Z_{\text {Tmid,3 }}$ & $Z_{T_{\max }}$ \\
\hline M2.0L & 0.82 & 0.85 & 0.88 & 0.93 & 0.97 \\
M1.5L & 0.81 & 0.85 & 0.90 & 0.95 & 0.98 \\
M2.0H & 0.65 & 0.75 & 0.80 & 0.90 & 0.98 \\
M1.5H & 0.63 & 0.70 & 0.80 & 0.90 & 0.98 \\
\hline
\end{tabular}

They correspond to different operating conditions and level of nonideality

\subsection{Schlieren images and pressure ratio}

For experiments at moderate non-ideal conditions, namely test M2.0L and M1.5L, top of Fig. 6 shows the nozzle geometry in non-dimensional coordinates, including the active pressure taps (where static pressure is measured). For test M2.0L, eight out of nine taps were active, while for test M1.5L only four taps were used, in the region of higher pressure gradients. In both cases, the geometrical throat is located at $x / H=10.29$. Schlieren images of the MDM vapor flow acquired at $t=0 \mathrm{~s}$ are also superimposed to the profile sketches.

Complex flow structures develop at the throat for test M2.0L (Fig. 6a) due to the presence of the recessed step, see Cozzi et al. (2015). The incoming flow rotates at the step edge through a first expansion fan (dark region at $x / H \approx 10.29$ ) and an oblique shock (thin dark line) is then formed at the reattachment point, downstream a local separation; the flow is finally rotated by a second fan (dark region at $x / H \approx 10.80$ ) at the connection between the step plateau and

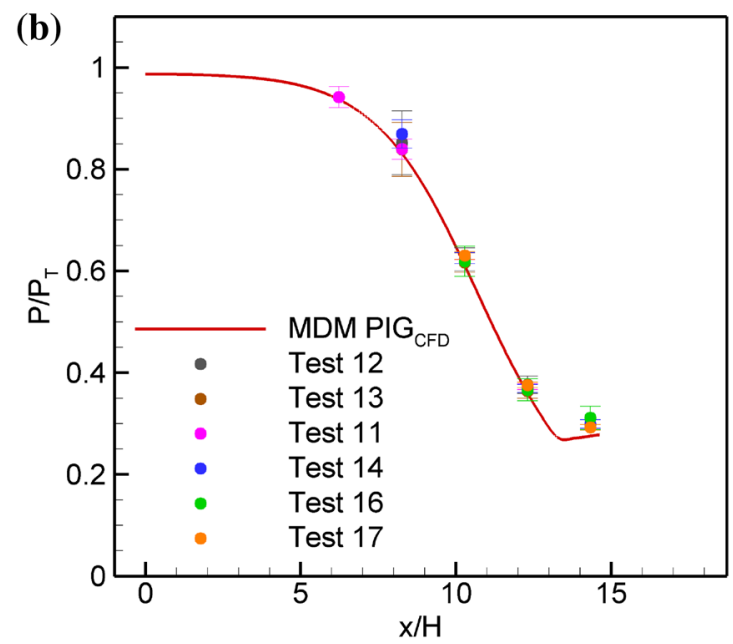

ence. a Pressure ratio $P / P_{\mathrm{T}}$ along the nozzle axis for tests on nozzle M2.0 at respective $Z_{T_{\max }}$. b Pressure ratio $P / P_{\mathrm{T}}$ along the nozzle axis for tests on nozzle M1.50 at respective $Z_{T_{\max }}$ 
(a)
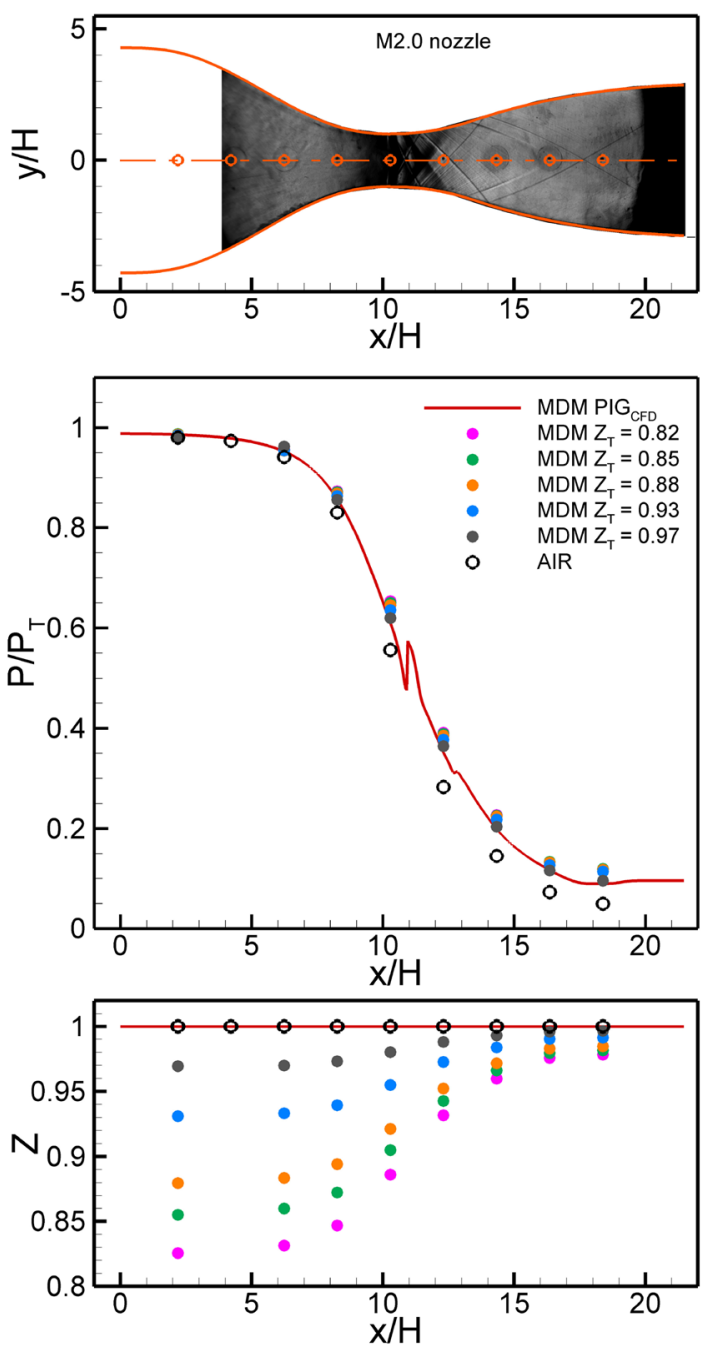

Fig. 6 Experimental results for test M2.0L (a) and M1.5L (b). Nozzle non-dimensional geometries (top) with overlapped schlieren images of the MDM vapor flow at time $t=0 \mathrm{~s}$, corresponding to $Z_{\mathrm{T}}=0.82$ (test M2.0L) and $Z_{\mathrm{T}}=0.81$ (test M1.5L). Throat is located at $x / H=10.29$. Experimental values at the axis of $P / P_{\mathrm{T}}$ (center) and

the contoured profile. Shock waves correctly appear as dark regions; expansions, which are expected to be visualized as bright fans, are instead dark. This is due to an overstep of the measuring range related to the high compressibility and the high density of the vapor flow. Indeed, this phenomenon attenuates as the test proceeds and expansion fans brighten as both compressibility and flow density reduce, see Conti et al. (2017). The flow at the axis is only locally perturbed by shock waves arising from the step. Furthermore, they form at low Mach number $(M \approx 1.2)$, and thus, they are weak enough to entail a negligible entropy production, despite well defined in the schlieren images. This was proven for the same geometry operated with air in Cozzi et al. (2015). The nozzle flow at the axis can be therefore assumed as (b)
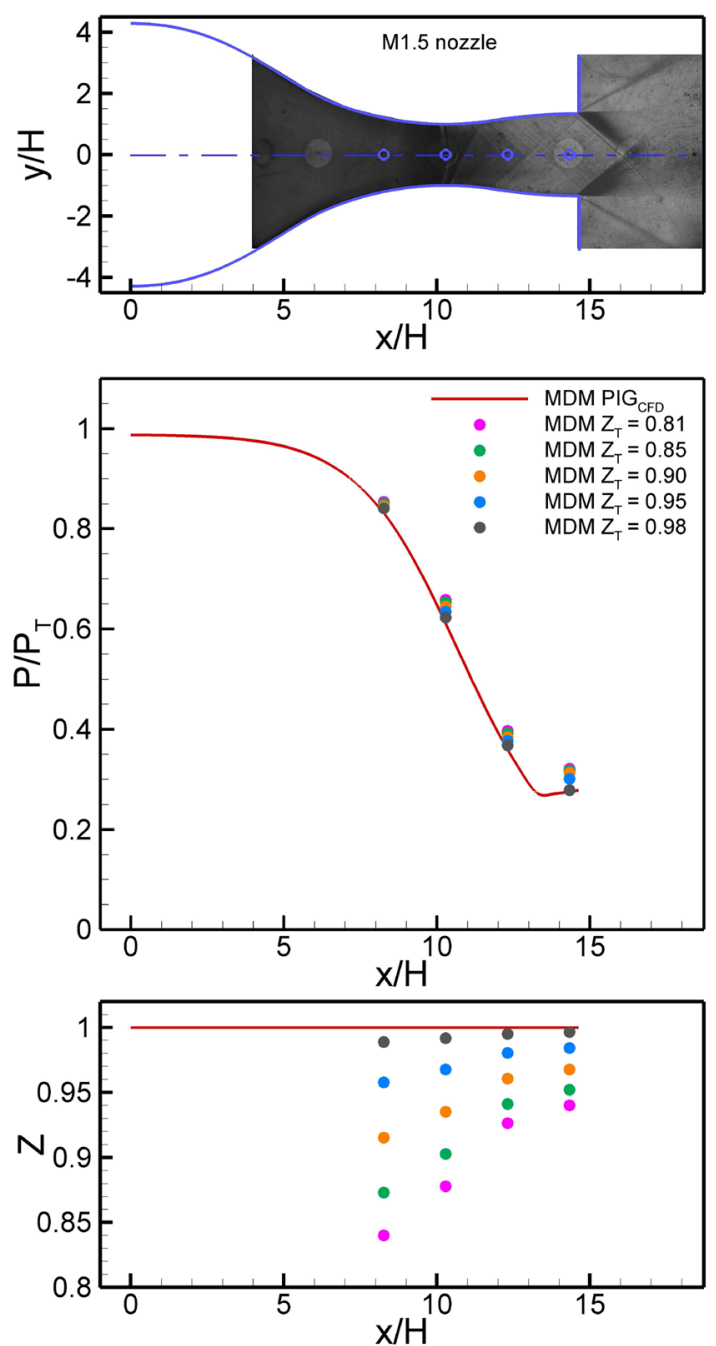

calculated $Z$ (bottom) as a function of the non-dimensional spatial coordinate $x / H$ at different $Z_{\mathrm{T}}$. CFD data from viscous calculation coupled with PIG model for MDM are superimposed to the experimental ones. For test M2.0L experimental points for the nozzle operated with air are included

isentropic, with a constant value of total pressure $P_{\mathrm{T}}$ at any selected time. From schlieren images, multiple weak shock reflections on the profiled walls are also clearly visible (at about $11.5<x / H<14.0$ ), as well as weak waves generated in the divergent portion by small perturbation of the flow due to profiled wall roughness. These are Mach waves and their slope, relative to the flow direction, represents a direct measurement of the local Mach number, see Sect. 5.2. Further details on the flow feature at the throat of nozzle M2.0 can be found in Spinelli et al. (2016) and Gori et al. (2017b).

Top of Fig. $6 \mathrm{~b}$ shows the geometry of nozzle M1.5 overlapped to schlieren images at time $t=0 \mathrm{~s}$ for test M1.5L. The profile is clean and exhibits at the same time an enhanced roughness at the contoured walls $(\mathrm{Ra} \approx 0.6 \mu \mathrm{m})$, to promote 
Mach waves formation, which are indeed well visible in the divergent portion of the nozzle. Consistently, the nozzle core flow is isentropic, as it is confirmed by the evidence from schlieren images that the flow is under-expanded. Indeed, for nozzle M1.5 the nozzle outlet section is optically accessible and the post-expansion is evidenced by the expansion fans and the slip lines visible at the nozzle exit. It is worth recalling here that, for all experimental runs, each nozzle operates in under-expanded conditions except at the test conclusion, when adapted and over-expanded conditions are reached.

The central part of Fig. 6 reports, for tests M2.0L (a) and $\mathrm{M} 1.5 \mathrm{~L}$ (b), the local pressure ratio $P / P_{\mathrm{T}}$ measured at the active taps at different $Z_{\mathrm{T}}$. For plot clarity, uncertainty

(a)
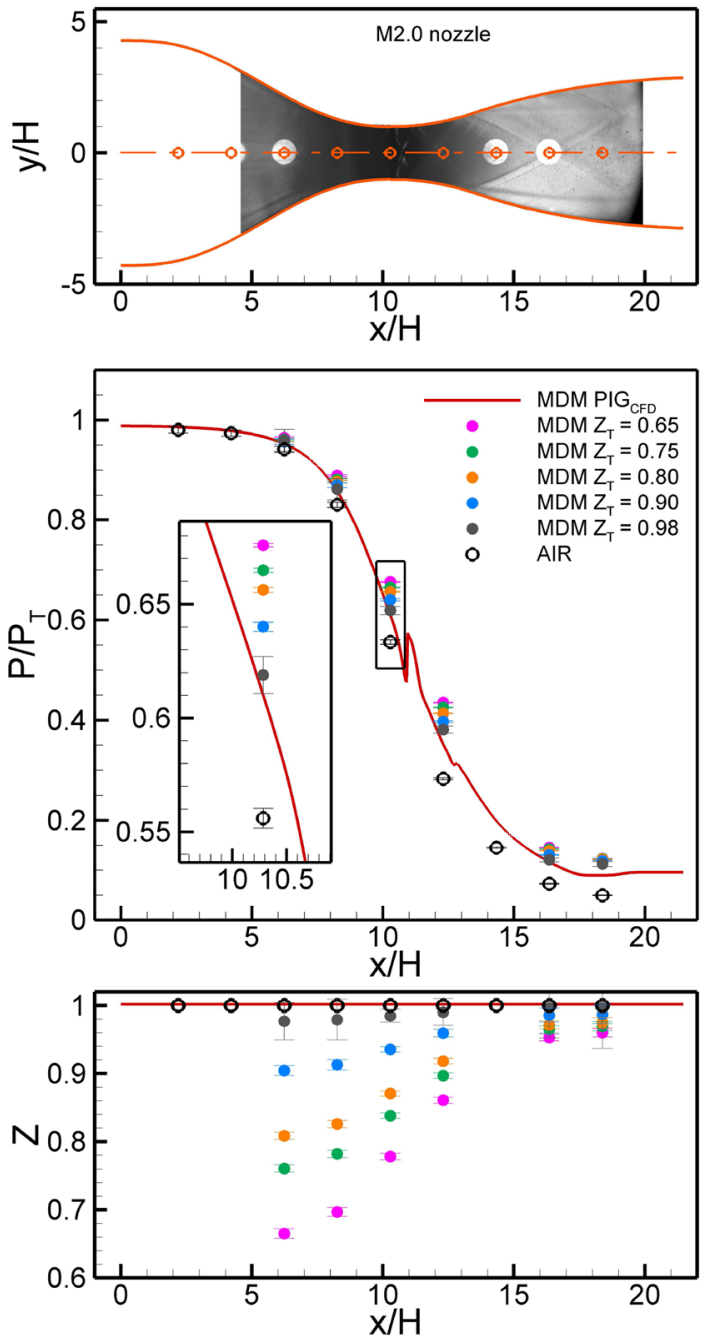

Fig. 7 Experimental results for test M2.0H (a) and M1.5H (b). Nozzle non-dimensional geometries (top) with overlapped schlieren images of the MDM vapor flow at time $t=4.75 \mathrm{~s}$, corresponding to $Z_{\mathrm{T}}=0.70$, for test $\mathrm{M} 2.0 \mathrm{H}$ and at time $t=1.60 \mathrm{~s}$, corresponding to $Z_{\mathrm{T}}=0.65$, for test M1.5H. Experimental values at the axis of $P / P_{\mathrm{T}}$ (center) and calculated $Z$ (bottom) as a function of the non-dimensional spatial coordinate $x / H$ at different $Z_{\mathrm{T}}$. CFD data from viscous bars are not reported here, but they are more significantly shown for experiments carried out at the highest level of non-ideality (see Fig. 7). As a reference, a continuous expansion profile is reported as extracted at the axis line from a two-dimensional CFD viscous calculation, performed by employing the SU2 software suite (Palacios et al. 2013; Vitale et al. 2015; Gori et al. 2017b) and by modeling MDM as a polytropic ideal gas. The constant specific heat ratio is $\gamma=1.018$, as obtained in the temperature range of interest for the presented experiments, according to Span (2000). Consistently, CFD results are well overlapped to data measured at maximum $Z_{\mathrm{T}}$, namely in the almost ideal gas state. For test M2.0L, two bumps are located at $x / H \approx 10.9$ and

(b)
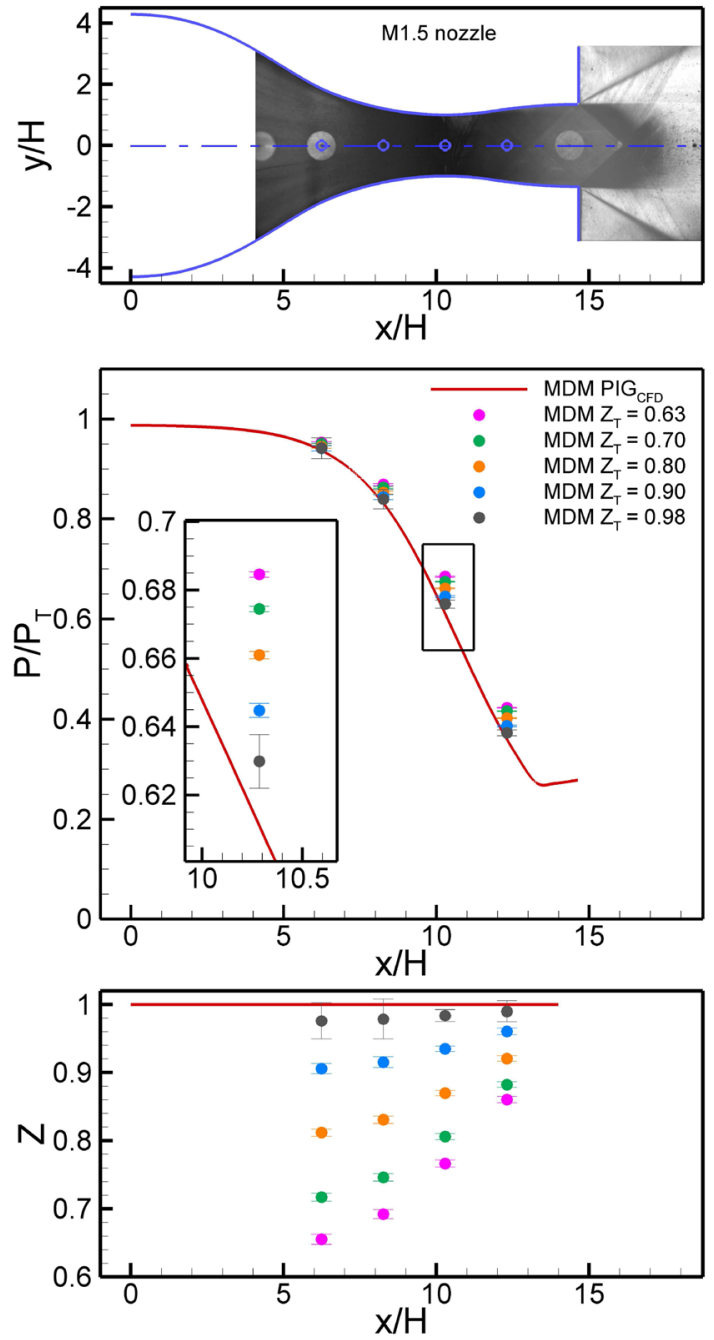

calculation coupled with PIG model for MDM are superimposed to the experimental ones. For test M2.0H experimental points for the nozzle operated with air are included. A detailed view of the measured pressure ratio is given at the throat $(x / H=10.29)$; both $P / P_{\mathrm{T}}$ data and calculated $Z$ trends are completed with the expanded uncertainty bars ( $95 \%$ confidence interval) 
$x / H \approx 12.7$, which are related to the shock wave originated at the step and to its first reflection. For nozzle M2.0 only, the local pressure ratio obtained by operating the nozzle with air is also reported; detailed results of these previous experiments with air are provided in Spinelli et al. (2015). Finally, the calculated values of the compressibility factor $Z$ along the two nozzle axis are plotted to identify departure from ideality along the entire expansion. For both tests performed with MDM, the value of $Z$ increases for the whole flow field as the extracted operating conditions become more ideal (increase of $Z_{\mathrm{T}}$ ); furthermore $Z$ increases as the expansion proceeds along the nozzle and approaches unity at the outlet. The rather trivial result of constant $Z=1$ is plotted for experiments performed with air (nozzle M2.0 only) and for CFD simulations with MDM treated as a perfect, namely, polytropic ideal, gas.

Similar to Figs. 6, 7 reports the nozzle profile sketches superimposed to schlieren images (top) at time $t=4.75 \mathrm{~s}$ (test $\mathrm{M} 2.0 \mathrm{H}$ ) and $t=1.60 \mathrm{~s}$ (test $\mathrm{M} 1.5 \mathrm{H}$ ), corresponding to $Z_{\mathrm{T}}=0.70$ and $Z_{\mathrm{T}}=0.65$ respectively, for the two experiments carried out in highly non-ideal conditions. Results in terms of pressure ratios (central) and compressibility factors (bottom) are also plotted. Six and four pressure taps are active for test $\mathrm{M} 2.0 \mathrm{H}$ and $\mathrm{M} 1.5 \mathrm{H}$, respectively. In both cases active pressure taps are located in the largest pressure gradient region. Schlieren images are considerably dark, especially at the throat region, where maximum density gradient is attained. This is caused by the above mentioned overstep of the measuring range, which is more evident than in the case of moderate non-ideal conditions and reaches its maximum at minimum $Z_{\mathrm{T}}$, see Conti et al. (2017). In fact, the flow features are scarcely visible in schlieren images at time $t=0 \mathrm{~s}\left(\right.$ minimum $\left.Z_{\mathrm{T}}\right)$ which are therefore not reported. Measuring range issues reduce as the test proceeds (and $Z_{\mathrm{T}}$ increases) and schlieren images gradually become more readable. However, the main flow features are qualitatively the same observed during low pressure tests, and similar considerations hold. Measured pressure ratio $P / P_{\mathrm{T}}$ and calculated compressibility factor $Z$ along the nozzle axis are plotted in the central and bottom parts of Fig. 7, together with uncertainty bars. Simulation results at the axis for MDM in polytropic ideal gas conditions and experimental results for nozzle M2.0 operated with air are also included. The overall vapor flow behavior is similar to the one obtained at moderate operating conditions, though non-ideal effects are higher as denoted by the lower level of $Z$ and the correspondingly higher local pressure ratios.

A first comparison on the pressure ratio distribution along the axis can be made between the nozzle M2.0 operated with air and with MDM at maximum $Z_{\mathrm{T}}(0.97$ for test M2.0L, 0.98 for test $\mathrm{M} 2.0 \mathrm{H}$ ), namely in almost ideal conditions. This allows to point out the effects on the expansion processes due to the different molecular complexity of the two fluids, that is, the different value of the specific heat ratio $\left(\gamma_{\mathrm{MDM}}=1.018, \gamma_{\text {air }}=1.4\right)$. At any axial coordinate the local pressure ratio is higher for MDM, due to its higher molecular complexity. This difference initially increases with the Mach number along the expansion, as compressibility effects start playing a role, while it starts reducing at higher Mach numbers (here above $M \approx 1.6-1.7$ for $\mathrm{MDM}$ ). This is in accordance with the well-known result that the pressure ratio tends to zero as Mach number tends to infinity. In addition, for a given nozzle, the pressure ratio is independent of the inlet condition, as confirmed by the comparison provided in Sect. 4.2 among different test at $Z_{T_{\max }}$, see Fig. 5. The described behavior is consistent with the one predicted by the quasi-one-dimensional theory for isentropic expanding flows of perfect gases (Thompson 1988) and it is not related to non-ideal effects.

To evidence non-ideal compressible flow effects, for each nozzle and operating condition with MDM, the expansion processes occurring at maximum $Z_{\mathrm{T}}$ are compared to those at lower $Z_{\mathrm{T}}$, up to the minimum value. For all the analyzed cases, the pressure ratio $P / P_{\mathrm{T}}$ along the expansion is higher than the ideal-gas one at $Z_{T_{\max }}$ and increases with increasing departure from ideality, as $Z_{\mathrm{T}}$ decreases. Such trend is ascribed to non-ideal effects and has no relation with measuring accuracy, since the uncertainty bars pertaining to different $Z_{\mathrm{T}}$ do not overlap. This is shown in Fig. 7 for tests at high pressure. It holds for all the performed tests and at almost any axial coordinate, with few exceptions in the initial converging portion of the nozzle and at high values of $Z_{\mathrm{T}}$. Indeed, in the initial converging region, higher full scale/uncertainty transducers are located; as a consequence, a higher uncertainty is obtained for pressure ratios, especially in the dilute gas state where the level of measured pressure is considerably low. Moreover, the initial part of the expansion features pressure ratios which are close to one and non-ideal compressible effects of different intensity are almost indistinguishable, despite marked by well separated values of $Z$ (see bottom of Fig. 7). Expanded uncertainty with a $95 \%$ confidence level for the pressure ratio was calculated by propagating the uncertainties of measured $P$ and $P_{\mathrm{T}}$, while for the compressibility factor a Monte Carlo method was applied (JCGM 2008a or JCGM 2008b), to propagate uncertainties of measured $P, P_{\mathrm{T}}$ and $T_{\mathrm{T}}$ in computed thermodynamic quantities such as $Z$ and $s$, which are calculated via the RefProp thermodynamic model. Note that no uncertainty in the thermodynamic model is accounted for.

Summarizing, for a given fluid and nozzle geometry, a dependence of the pressure ratio on the inlet stagnation conditions is documented here for the first time, which proves the non-ideal nature of the flow in all the analyzed cases. Indeed, this is consistent with the non-ideal behavior of quasi-one-dimensional isentropic expansions predicted by Tsien (1946). The described trend of measured pressure 
ratio is qualitatively in agreement with the one predicted by the van der Waals model applied to a quasi-one-dimensional nozzle expansion of MDM vapor, while a very good quantitative consistence is found with the results of a twodimensional viscous CFD calculation (Gori et al. 2017b) implementing highly accurate thermodynamic models for MDM, such as RefProp model or the improved Peng Robinson Stryjek Vera (iPRSV) equation of state (van der Stelt et al. 2012). The dependence of the flow field on the inlet conditions is found to be not negligible, for both cases of moderate and high deviations from the ideal-gas state. For example, at the geometrical throat, the deviation of $P / P_{\mathrm{T}}$ with respect to the one obtained at $Z_{T_{\max }}=0.98$ is of about $5.6 \%$ for $Z_{\mathrm{T}}=0.81$ (test $\mathrm{M} 1.5 \mathrm{~L}$ ), and of about $8.7 \%$ for $Z_{\mathrm{T}}=0.63$ (test $\left.\mathrm{M} 1.5 \mathrm{H}\right)$.

To assess if non-ideal nozzle flow is influenced by the full stagnation state, namely $P_{\mathrm{T}}$ and $T_{\mathrm{T}}$, or by the stagnation compressibility factor $Z_{\mathrm{T}}$ only, different nozzle flows exhibiting the same $Z_{\mathrm{T}}$ but different total conditions are compared. Figure 8 shows the result of this comparison in terms of pressure ratio for nozzle M1.5 at moderate level of nonideality $\left(Z_{\mathrm{T}}=0.85\right)$, where a sufficiently large data set was available in a reasonably wide range of nozzle operating conditions. The nozzle flow at the axis appears to be mainly dependent on the compressibility factor $Z_{\mathrm{T}}$ rather than on the full total state, and this trend seems to be preserved not only at higher $Z_{\mathrm{T}}$, as expected, but also at higher level of non-ideality up to $Z_{\mathrm{T}}$ as low as 0.70 . However, the data set available for comparison is not adequately large and spans a range of operating conditions (at any $Z_{\mathrm{T}}$ ) not sufficiently

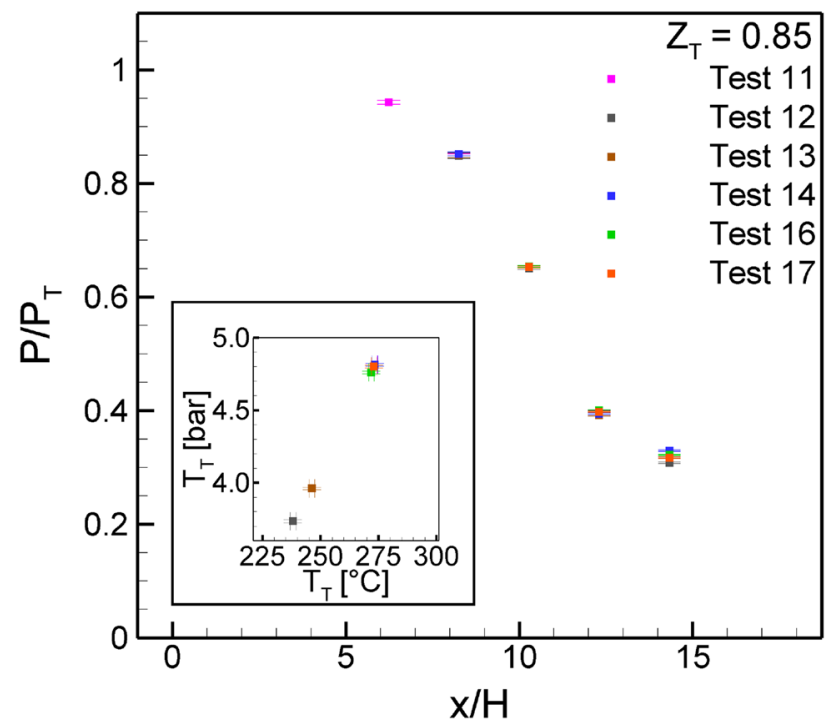

Fig. 8 Comparison of pressure ratio $P / P_{\mathrm{T}}$ distribution along the nozzle axis for tests $11,12,13,14,16$, and 17. Nozzle inlet stagnation conditions $P_{\mathrm{T}}$ and $T_{\mathrm{T}}$ are also reported for the data sets which are compared at the same total compressibility factor $Z_{\mathrm{T}}=0.85$ wide to draw general conclusions, especially at highly nonideal states. In this respect, to evaluate more accurately the role of inlet compressibility factor in non-ideal nozzle flows, a dedicated experimental campaign is currently underway on the TROVA facility.

\subsection{Mach number}

In the four analyzed tests, the flow Mach number $M$ was directly measured along the axis of the nozzle diverging portion from schlieren visualizations. The measurement is performed through the evaluation of the slope of Mach waves, identified within the schlieren images extracted at the same $Z_{\mathrm{T}}$ selected for the analysis of pressure ratios. At the axis position where pressure taps are located, the measured Mach numbers are also compared with those calculated as a function of measured pressure and total conditions, by applying the isentropic hypothesis and to the RefProp model, namely $M=u / c$ where $u$ is the velocity modulus and $c$ is the speed of sound. Both $u$ and $c$ are computed from the local measured value of the pressure and from stagnation conditions.

Mach waves are infinitely weak (thus isentropic) oblique waves, originated by the roughness at the profiled nozzle walls. As such, they are present only within the supersonic flow region in the divergent portion of the nozzle and feature density gradients which can be observed using schlieren visualization, which allows for a larger number of measuring points at the axis with respect to pressure data. The slope $\mu$ of the Mach waves with respect to the local flow angle depends only on the local value of the Mach number via the relation $M=\frac{1}{\sin (\mu)}$. By virtue of symmetry, the flow velocity at the centerline is parallel to the axis, and $\mu$ is simply the angle formed between the wave and the nozzle axis itself. The detection of Mach lines from schlieren images is a powerful method to obtain a direct measurement of the local Mach number without involving any thermodynamic model in the calculation, if the direction of the velocity is known.

Results obtained for Mach number measurements are presented here referring to the nozzle M1.5 only. For this nozzle, Mach lines are more clearly visible, due to the improved profile roughness (see Sect. 2), which enhances the wave contrast, and due to the absence of any well defined structure (shocks/reflected shocks/expansion fans) originated from step singularities, which inevitably overlap with weak Mach waves for nozzle M2.0. Top of Fig. 9 depicts an enlarged view of the divergent portion of the nozzle which clearly visualizes the dark lines originated at the contoured walls. The overlapped lines mark the Mach waves identified at the axis by processing the images recorded with a line detection algorithm, implementing the Hough transform (Duda and Hart 1972) and the line detection method proposed in Lo and Tsai (1995). The schlieren image is first cut into sub-images 
(a)
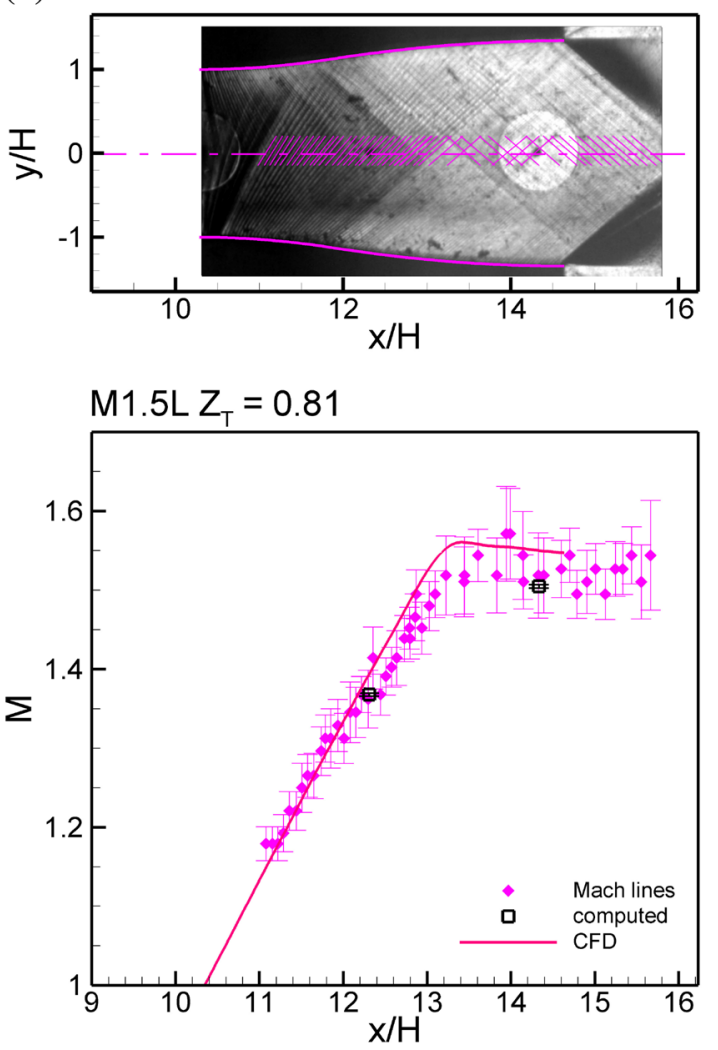

Fig. 9 Schlieren images (top) and measured Mach number distribution along the axis (bottom) for the divergent portion of the nozzle and for tests M1.5L (a) at $Z_{T}=0.81$ and test M1.5H (b) at $Z_{T}=0.65$. Mach number was measured through the slope of Mach waves identi-

centered on the axis. The gray-scaled images are then binarized and the standard Hough transform is performed. Peaks of the Hough transform are detected, each corresponding to a line in the image. The correct slope $\mu$ of each line is finally found according to the algorithm illustrated in Lo and Tsai (1995).

The angular resolution is limited by the size of image pixels. Once one line is identified, the angular expanded uncertainty $U_{\mu}(95 \%$ confidence level) is computed by assuming a uniform probability distribution within the pixel of the end point location (JCGM 2008). The bottom part of Fig. 9 reports the Mach number trend along the nozzle axis, together with the corresponding uncertainty bars. The two plots are obtained by applying the described method to schlieren images extracted from tests $\mathrm{M} 1.5 \mathrm{~L}$ and $\mathrm{M} 1.5 \mathrm{H}$ at the minimum value of $Z_{\mathrm{T}}$ at which good-quality images were available. In particular, at the lowest $Z_{\mathrm{T}}=0.63$, density gradients are beyond the schlieren measuring range and the resulting image is almost completely dark in the throat region. Therefore, for test $\mathrm{M} 1.5 \mathrm{H}$, condition $Z_{\mathrm{T}}=0.65 \mathrm{was}$ used. (b)

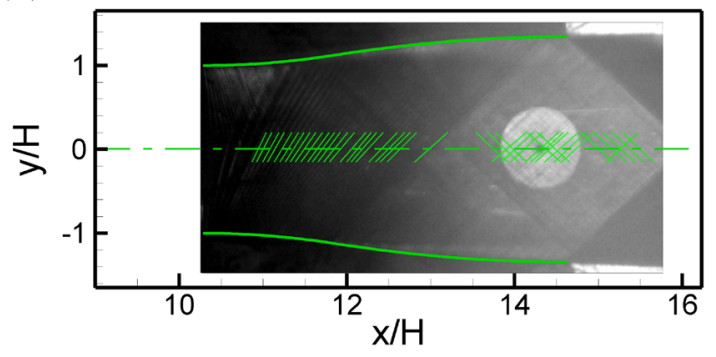

$\mathrm{M} 1.5 \mathrm{H} \mathrm{Z} \mathrm{T}_{\mathrm{T}}=0.65$

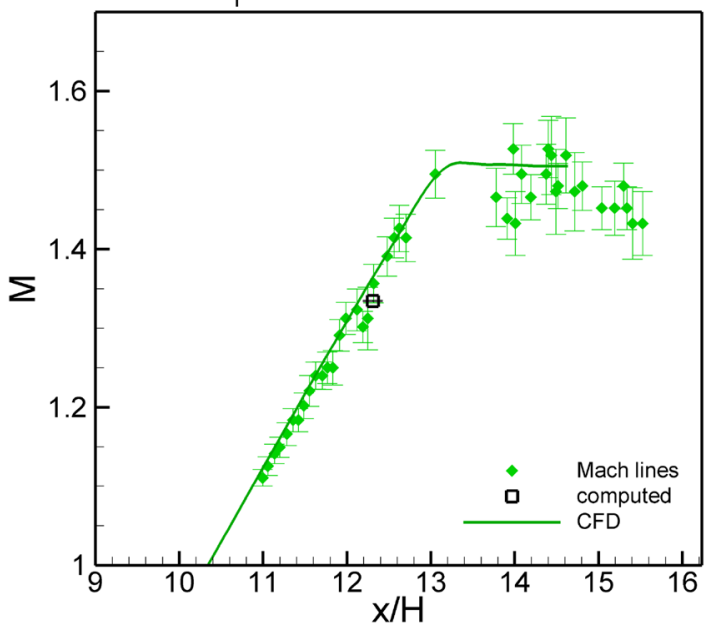

fied in the schlieren images and marked here (top) with overlapped purple (a) and green (b) lines. The geometry of the divergent profile of nozzle M1.5 is also superimposed to the schlieren images

As clearly pointed out by Fig. 9, the uncertainty $U_{\mathrm{M}}$ of Mach measurement increases with Mach number, given the angular uncertainty $U_{\mu}$, which is related to the sub-image dimension and to the camera resolution. Indeed, the uncertainty propagated to Mach number is as follows:

$U_{\mathrm{M}}=M \sqrt{M^{2}-1} U_{\mu}$

where the term $M \sqrt{M^{2}-1}$ increases almost quadratically with Mach number above $M \approx 1.2$. Therefore, the increased uncertainty is due to the dependence of the Mach number on the Mach line slope $\mu$, through the inverse sine function. At small supersonic Mach numbers $\mu$ is close to $90^{\circ}$ and the function $\sin ^{-1}$ is almost insensitive to angular variation related to uncertainty; conversely, its sensitivity to angular changes increases with Mach number especially at high $M$ where the trend is strongly non-linear. This entails an increasing uncertainty, in particular above $M=1.5$. In other words, for a small perturbation occurring in highly supersonic flows, a significant acceleration is required to produce non-negligible sharpening of the Mach surface, whose local semi-aperture is $\mu$. 
In Fig. 9 a comparison is also given between the measured Mach and the ones calculated by using pressure and temperature measurements $M=M\left(P, s\left(P_{\mathrm{T}}, T_{\mathrm{T}}\right)\right)$, through the RefProp model. Results are in good accordance (also for test and conditions not reported in the figure), thus confirming the accuracy of the thermodynamic model employed. The Mach distribution at the axis as extracted from a two-dimensional, viscous CFD calculation is also plotted for reference.

Figure 10 contains a comparison between the local Mach number measured at the nozzle axis at $Z_{\mathrm{T}}=0.65$ (test $\mathrm{M} 1.5 \mathrm{H}$ ) and $Z_{\mathrm{T}}=0.90$ test $(\mathrm{M} 1.5 \mathrm{~L})$. These values were chosen as the minimum and the maximum compressibility factors at which schlieren images quality is good enough to allow the detection of Mach lines. The comparison shows that as the non-ideal effects increase, the Mach value level decreases with respect to its perfect gas counterpart. Although a partial overlap of uncertainty bars is present, especially at the expansion end, where the exit conditions are reached, the trend is clearly visible, in particular, within the region of higher gradients, up to $M \approx 1.55$. This represents a further proof of the dependence of the nozzle flow field on the stagnation conditions, for a given geometry and working fluid. In addition, the trend is consistent with the one found for pressure ratio (see Figs. 6 and 7) and it is confirmed for intermediate values of $Z_{\mathrm{T}}$, not reported here for plot clarity. For a given nozzle geometry, the reduction of supersonic Mach number with increasing non-ideality of the flow is also predicted by the one-dimensional theory. Indeed, for an isentropic nozzle flow, the Mach number gradient along the axial coordinate $x$ reads

$\frac{1}{M} \frac{\mathrm{d} M}{\mathrm{~d} x}=\frac{1+(\Gamma-1) M^{2}}{M^{2}-1} \frac{1}{A} \frac{\mathrm{d} A}{\mathrm{~d} x}$,

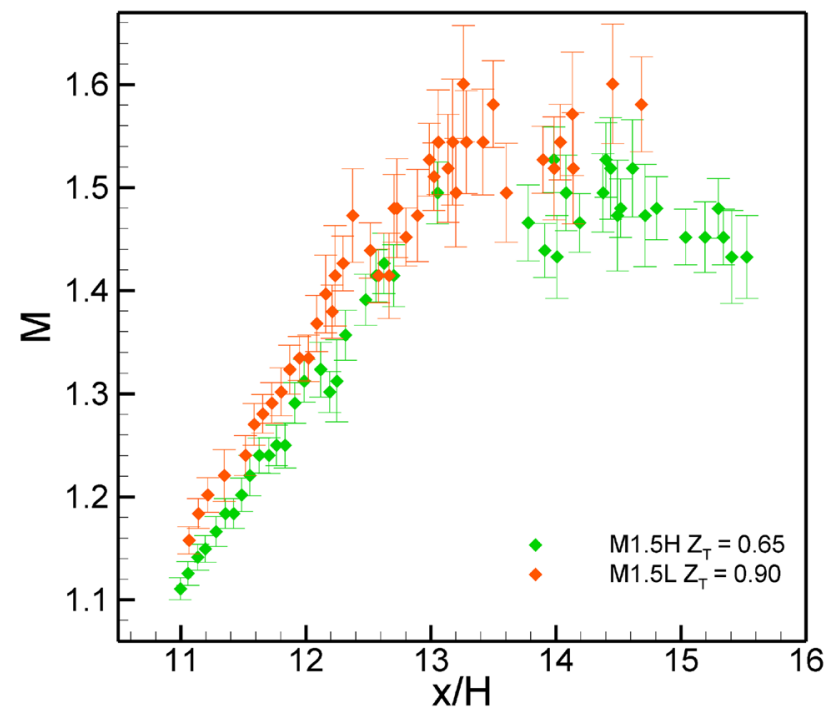

Fig. 10 Comparison between measured Mach number distribution at the axis of the nozzle diverging portion for tests M1.5L at $Z_{\mathrm{T}}=0.65$ and for test $\mathrm{M} 1.5 \mathrm{H}$ at $Z_{\mathrm{T}}=0.90$ where $A=A(x)$ is the value of the cross-sectional area along the axis, see Thompson (1991). As the level of non-ideality increases, the value of $\Gamma$ reduces, entailing a lower gradient of Mach number along the diverging portion, and thus, a lower Mach number is attained at any axial coordinate. Finally, CFD simulation performed also gave consistent results and well-comparable trends, see Gori et al. (2017b).

\section{Conclusion}

Results of an experimental campaign carried out to study non-ideal compressible flows of high molecular complexity vapors in supersonic nozzles are presented here for the first time. Nozzle expansion processes were selected as the most significant ones, for both fundamental research in non-ideal compressible fluid dynamics and industrial applications, especially for ORC power systems. A correct modeling of such unconventional fluid flows, by either theoretical or computational tools, demands detailed and reliable experimental data, which were still not available in the open literature, also due to difficulties of performing accurate measurements in high temperature and potentially condensing vapor flows. The reported measurements represent the first results obtained, aiming at overcoming the lack of experimental data and providing a reference data set.

Several tests were performed on the nozzle test section of the Test Rig for Organic VApors (TROVA), at the Laboratory of Compressible fluid-dynamics for Renewable Energy Application (CREA Lab) of Politecnico di Milano, Italy. The fluid investigated was siloxane MDM, which is particularly suited for the proposed study, due to both its high molecular complexity and its wide application on ORC power systems. Experiment repeatability was thoroughly verified by comparing several tests performed at different operating conditions in the ideal-gas limit and a few tests carried out in comparable non-ideal states. The measuring procedure revealed reliable and the methodology applied allowed to achieve a satisfactory low uncertainty level for the measured data.

Two planar and converging-diverging nozzles were operated with MDM vapor, from highly non-ideal conditions, including thermodynamic regions of industrial interest, to almost ideal ones. The flow field was investigated in terms of pressure distribution at the axis, of inlet total temperature and pressure and of schlieren visualization, which provided a direct measurement of the Mach number at the centerline of the nozzle diverging portion. Different measured quantities exhibit mutually consistent trend and calculations performed by applying the RefProp thermodynamic model to the explored operating conditions support the congruence of data. The analysis is based on local pressure ratio $P / P_{\mathrm{T}}$ and Mach number measurements along the nozzle axis, and it is therefore carried out without resorting to any computational 
model. Some results of numerical calculation were reported here only to support the experimental results.

The nozzle flow is proven to be non-ideal, since the inlet thermodynamic conditions significantly affect both the pressure ratio and the Mach number distribution along the nozzle axis, contrarily to the well-known behavior of perfect gases. Hence, the present study confirms the need of accounting for non-ideal effects in the design and analysis of component operating in the non-ideal compressible regime of molecularly complex vapors. In addition, the measurement techniques selected proven their suitability in detecting non-ideal behaviors in compressible flows. A reliable methodology is, therefore, reported, which is applicable to perform experiments on high temperature and potentially condensing vapor flows in highly nonideal thermodynamic conditions. This allows to provide the demanded experimental data and to establish reference test cases for non-ideal compressible flows.

Acknowledgements The research is funded by the European Research Council under ERC Consolidator Grant 2013, project NSHOCK 617603. The initial layout of the TROVA plant was funded by Turboden S.r.l. The authors wish to thank Camilla Conti for her valuable help.

Open Access This article is distributed under the terms of the Creative Commons Attribution 4.0 International License (http://creativeco mmons.org/licenses/by/4.0/), which permits unrestricted use, distribution, and reproduction in any medium, provided you give appropriate credit to the original author(s) and the source, provide a link to the Creative Commons license, and indicate if changes were made.

\section{References}

Antonini C, Persico G, Rowe AL (2008) Prediction of the dynamic response of complex transmission line system for unsteady pressure measurement. Meas Sci Technol 19(12):125401

Colonna P, Guardone A (2006) Molecular interpretation of nonclassical gasdynamics of dense vapors under the van der Waals model. Phys Fluids 18(5):056,101-1-14

Colonna P, Guardone A, Nannan R (2007) Siloxanes: a new class of candidate Bethe-Zel'dovich-Thompson fluids. Phys Fluids 19(8):086,102-1-12

Colonna P, Harinck J, Rebay S, Guardone A (2008) Real-gas effects in organic rankine cycle turbine nozzles. J Propuls Power 24(2):282-294

Colonna P, Casati E, Trapp C, Mathijssen T, Larjola J, TurunenSaaresti T, Uusitalo A (2015) Organic Rankine cycle power systems: from the concept to current technology, applications, and an outlook to the future. ASME J Eng Gas Turbines Power 137:100,801-1-19

Conti C, Spinelli A, Guardone A, Cozzi F, Cammi G, Zocca M (2017) Schlieren visualization of non-ideal compressible fluid flows. In: 12th international conference on heat transfer, fluid mechanics and thermodynamics, HEFAT 2017

Cozzi F, Spinelli A, Carmine M, Cheli R, Zocca M, Guardone A (2015) Evidence of complex flow structures in a convergingdiverging nozzle caused by a recessed step at the nozzle throat.
In: 45th AIAA fluid dynamics conference, Dallas, 22-26 June 2015

Cramer MS, Fry RN (1993) Nozzle flows of dense gases. Phys Fluids 5(5):1246-1259

Duda RO, Hart PE (1972) Use of the Hough transformation to detect lines and curves in pictures. Graph Image Process 15(1):11-15

Gori G, Vimercati D, Guardone A (2017a) Non-ideal compressible-fluid effects in oblique shock waves. J Phys Conf Ser 821(1):012003. http://stacks.iop.org/1742-6596/821/i=1/ $\mathrm{a}=012003$

Gori G, Zocca M, Cammi G, Spinelli A, Guardone A (2017b) Experimental assessment of the open-source SU2 CFD suite for ORC applications. Energy Proc 129(Suppl C):256-263. http://www. sciencedirect.com/science/article/pii/S1876610217340171. In: 4th international seminar on ORC power systems, 13-15th Sep 2017. Politecnico di Milano Bovisa Campus Milano, Italy

Guardone A, Vimercati D (2016) Exact solutions to non-classical steady nozzle flows of Bethe-Zel'dovich-Thompson fluids. J Fluid Mech 800:278-306

Guardone A, Spinelli A, Dossena V (2013) Influence of molecular complexity on nozzle design for an organic vapor wind tunnel. ASME J Eng Gas Turbines Power 135(042):307

Hung PC, Irwin G, Kee R, McLoone S (2005) Difference equation approach to two-thermocouples sensor characterization in constant velocity flow environment. Rev Sci Instrum 76:024902

JCGM (2008a) JCGM 101: evaluation of measurement data-Supplement I to the 'Guide to the expression of uncertainty in measurement'-Propagation of distributions using Monte Carlo method. Tech. rep., JCGM

JCGM (2008b) JCGM 100: evaluation of measurement data-guide to the expression of uncertainty in measurement. In: Technical report, JCGM

Kluwick A (2004) Internal flows of dense gases. Acta Mech 169:123-143

Lemmon EW, Huber ML, McLinden MO (2013) NIST standard reference database 23: reference fluid thermodynamic and transport properties-REFPROP, version 9.1. National Institute of Standards and Technology. https://www.nist.gov/srd/refprop

Lo RC, Tsai WH (1995) Gray-scale Hough trasform for thick line detection in grey-scale images. Pattern Recognit 28:647-661

Macchi E, Astolfi M (2017) Organic Rankine cycle (ORC) power systems. In: Technologies and applications. Woodhead publishing series in energy: number 107. Elsevier, New York

Palacios F, Colonno MR, Aranake AC, Campos A, Copeland SR, Economon TD, Lonkar AK, Lukaczyk TW, Taylor TWR, Alonso JJ (2013) Stanford University Unstructured ( $\left.\mathrm{SU}^{2}\right)$ : an open-source integrated computational environment for multi-physics simulation and design. In: AIAA paper 2013-0287 51st AIAA aerospace sciences meeting and exhibit

Pini M, Spinelli A, Dossena V, Gaetani P, Casella F (2011) Dynamic simulation of a test rig for organic vapours. In: Proceedings of 5th conference on energy sustainability, ASME EsFuelCell2011, Washington

Span R (2000) Multiparameter equations of state: an accurate source of thermodynamic property data. Springer, Berlin

Span R, Wagner W (2003) Equations of state for technical applications. I. Simultaneously optimized functional forms for nonpolar and polar fluids. Int J Thermophys 24(1):1-39

Spinelli A, Dossena V, Gaetani P, Osnaghi C, Colombo D (2010) Design of a test rig for organic vapours. In: Proceedings of ASME turbo expo, Glasgow

Spinelli A, Pini M, Dossena V, Gaetani P, Casella F (2013) Design, simulation, and construction of a test rig for organic vapours. ASME J Eng Gas Turbines Power 135(042):303

Spinelli A, Guardone A, Cozzi F, Carmine, Cheli R, Zocca M, Gaetani P, Dossena V (2015) Experimental observation of non-ideal 
nozzle flow of siloxane vapor MDM. In: 3rd international seminar on ORC power systems, vol 103

Spinelli A, Cozzi F, Dossena V, Gaetani P, Zocca M, Guardone A (2016) Experimental investigation of a non-ideal expansion flow of siloxane vapor MDM. In: Proceedings of ASME turbo expo, Seoul, vol 3

van der Stelt T, Nannan N, Colonna P (2012) The IPRSV equation of state. Fluid Phase Equilib 330:24-35

Thompson PA (1971) A fundamental derivative in gas dynamics. Phys Fluids 14(9):1843-1849

Thompson PA (1988) Compressilbe fluid dynamics. McGraw-Hill, New York

Thompson PA (1991) Liquid-vapor adiabatic phase changes and related phenomena. In: Kluwick A (ed) Nonlinear waves in real fluids. Springer, New York, pp 147-213

Thompson PA, Lambrakis KC (1973) Negative shock waves. J Fluid Mech 60:187-208
Tsien HS (1946) One-dimensional flows of a gas characterized by vander Waal's equation of state. J Math Phys 25(1-4):301-324

Vimercati D, Gori G, Spinelli A, Guardone A (2017) Non-ideal effects on the typical trailing edge shock pattern of ORC turbine blades. Energy Proc 129(Suppl C):1109-1116. http://www.sciencedirect. com/science/article/pii/S1876610217341498. In: 4th international seminar on ORC power systems, 13-15th Sep 2017. Politecnico di Milano Bovisa Campus Milano, Italy

Vitale S, Gori G, Pini M, Guardone A, Economon TD, Palacios F, Alonso JJ, Colonna P (2015) Extension of the SU2 open source CFD code to the simulation of turbulent flows of fluids modelled with complex thermophysical laws. In: 22nd AIAA computational fluid dynamics conference, AIAA paper 2760

Publisher's Note Springer Nature remains neutral with regard to jurisdictional claims in published maps and institutional affiliations. 